
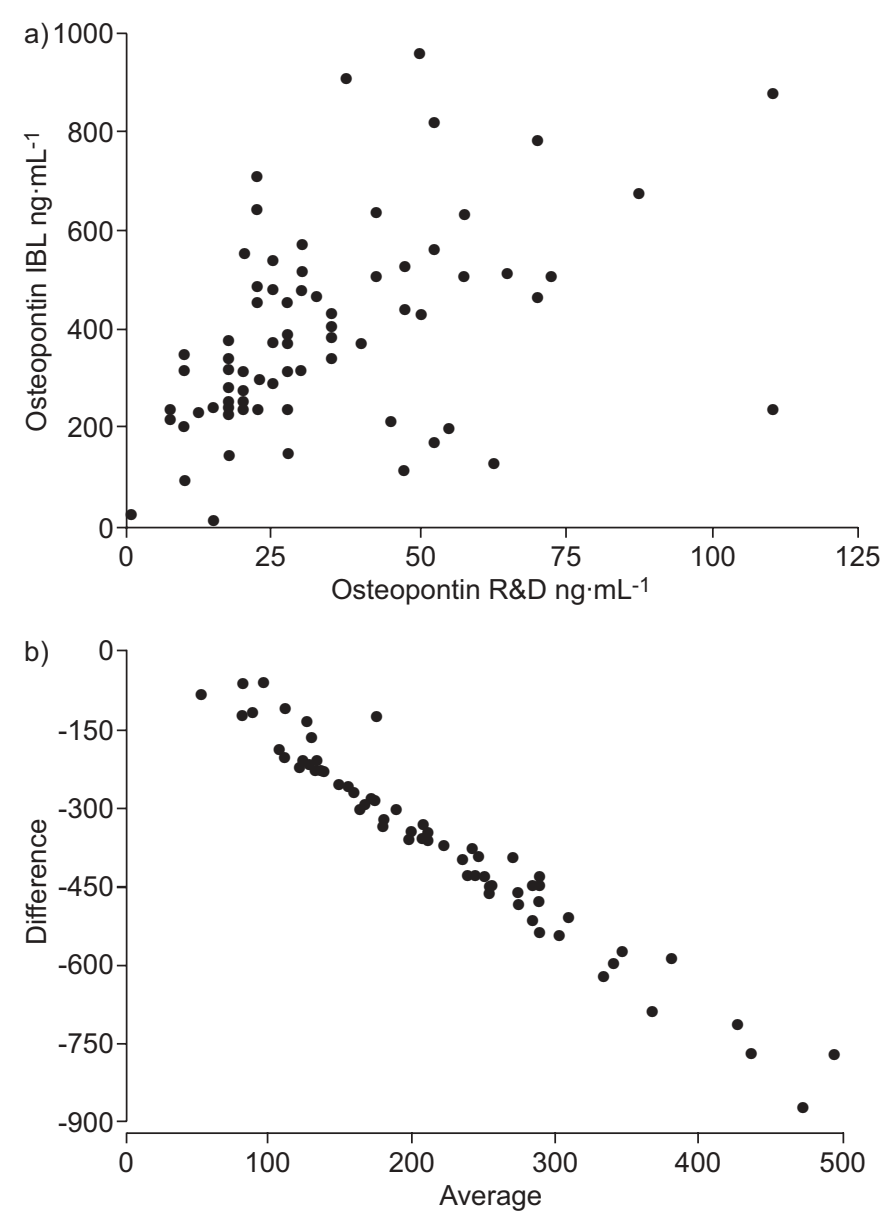

FIGURE 1. a) Correlation between the values obtained for osteopontin in plasma assayed with the Quantikine Human Osteopontin Immunoassay (R\&D systems, Minneapolis, MN, USA) (R\&D) and the Human osteopontin ELISA (Immuno-Biological Laboratories Co., Ltd, Gunma, Japan) (IBL). Spearman $\rho=0.4924$. b) Bland-Altman plot of the measurements for osteopontin showing a strong proportional error between the two methods.

in which direction. Mainly, both assays measure the same target molecule despite using different antibodies. These data must be taken into account when interpreting and comparing the results between different publications as well as choosing an assay for use in practice.
D. Constantinescu*, M. Vornicu" , C. Grigoriu ", C. Cozmei* and B.D. Grigoriu ${ }^{\#+}$

*Institute of Public Health, "Clinic of Pulmonary Diseases, "Depts of Forensic Pathology, and ${ }^{+}$Dept of Pulmonary Diseases, University of Medicine and Pharmacy "Gr. T. Popa", Iasi, Romania.

Correspondence: B.D. Grigoriu, Dept of Pulmonary Diseases, University of Medicine and Pharmacy "Gr. T. Popa" Iasi, Str Universitatii 16, 700115 Iasi, Romania. E-mail: b_grigoriu@ hotmail.com

Support Statement: This research has been funded by the National Council for Scientific Research in Higher Education (CNCSIS) by the grant number 1191/2007.

Statement of Interest: None declared.

\section{REFERENCES}

1 Isa S-I, Kawaguchi T, Teramukai S, et al. Serum osteopontin levels are highly prognostic for survival in advanced non-small cell lung cancer: results from JMTO LC 0004. J Thorac Oncol 2009; 4: 1104-1110.

2 Inomata S, Shijubo N, Kon S, et al. Circulating interleukin-18 and osteopontin are useful to evaluate disease activity in patients with tuberculosis. Cytokine 2005; 30: 203-211.

3 Pardo A, Gibson K, Cisneros J, et al. Up-regulation and profibrotic role of osteopontin in human idiopathic pulmonary fibrosis. PLoS Med 2005; 2: e251.

4 Chung KF, Adcock IM. Multifaceted mechanisms in COPD: inflammation, immunity, and tissue repair and destruction. Eur Respir J 2008; 31: 1334-1356.

5 Scherpereel A, Astoul P, Baas P, et al. Guidelines of the European Respiratory Society and the European Society of Thoracic Surgeons for management of malignant pleural mesothelioma. Eur Respir J 2010; 35: 479-495.

6 Creaney J, Yeoman D, Demelker Y, et al. Comparison of osteopontin, megakaryocyte potentiating factor, and mesothelin proteins as markers in the serum of patients with malignant mesothelioma. J Thorac Oncol 2008; 3: 851-857.

7 Vordermark D, Said HM, Katzer A, et al. Plasma osteopontin levels in patients with head and neck cancer and cervix cancer are critically dependent on the choice of ELISA system. BMC Cancer 2006; 6: 207.

8 Bland JM, Altman DG. Statistical methods for assessing agreement between two methods of clinical measurement. Lancet 1986; 1: 307-310.

DOI: $10.1183 / 09031936.00160609$

\title{
Venoatrial compression by lymphadenopathy in sarcoidosis
}

To the Editors:

Thoracic lymphadenopathy in sarcoidosis usually displays characteristic and well-known features, such as a symmetrical hilar distribution and a noncompressive nature. However, compressive lymphadenopathy is known as an atypical yet possible presentation of the disease. Several case reports and reviews have previously reported compressions of lobar or segmental bronchi, pulmonary artery branches, superior vena cava or the left laryngeal recurrent nerve [1-4]. To the best of 

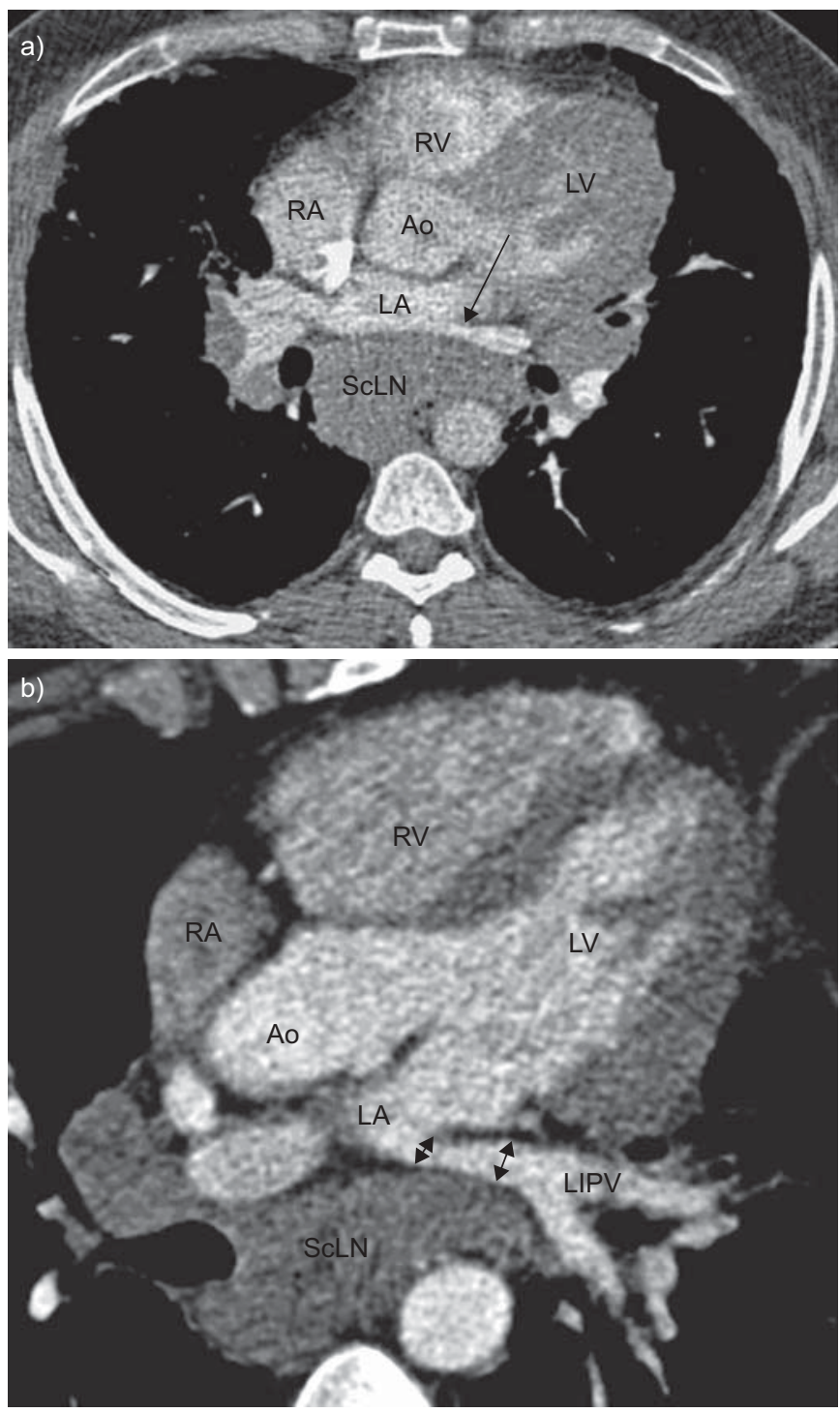

FIGURE 1. a) Contrast-enhanced computed tomography scan in a male with sarcoidosis showing left atrial compression (LA) and anteroposterior stenosis of the left inferior pulmonary vein (LIPV; arrow) caused by enlarged subcarinal lymph nodes $(\mathrm{ScLN})$. b) In the same patient, oblique reformatted image confirmed the stenosis of the LIPV (arrow). LV: left ventricle; RA: right atrium; RV: right ventricle;

our knowledge, compression of the heart has never been described. We report here the clinical, morphological and functional characteristics of four cases of sarcoidosis with venoatrial compression caused by voluminous subcarinal lymph nodes. The diagnosis of sarcoidosis had been made according to the American Thoracic Society (ATS)/European Respiratory Society (ERS)/World Association of Sarcoidosis and other Granulomatous Disorders (WASOG) criteria [5]. One patient had stage one disease on diagnosis (Löfgren's syndrome) and eventually evolved to stage two. Three patients had stage two sarcoidosis; one of them displayed characteristic skin involvement, another one cervical lymphadenopathy and possible muscular involvement. In the third patient, disease was limited to the thorax, and evolved into stage four during follow-up.
Venoatrial compression became apparent on computed tomography (CT) scans performed during follow-up. All patients complained of increased shortness of breath on exercise at that time. CT scan (fig. 1) showed enlarged subcarinal lymphadenopathy, compressing the left atrial posterior wall, giving it a straight or convex shape. Extrinsic compression of one or several pulmonary veins was also noticed for three patients, inducing stenosis of up to $50 \%$ (table 1). Parenchymal involvement of sarcoidosis was also increased in all patients, but remained moderate for patients one and four. For these two patients, the pulmonary functional tests at rest remained normal, while the two other patients (two and three) exhibited a restrictive pattern with significant alteration of carbon monoxide diffusion. All patients had significant alteration of aerobic capacity (table 1). Both trans-thoracic and trans-parietal echocardiographies were performed in three patients (one, two and four), and did not reveal pulmonary hypertension but showed left atrial compression. The interatrial septum was deviated towards the right atrium, indicating increased left atrial pressure. The left atrium volume was $15 \mathrm{~mL} \cdot \mathrm{m}^{-2}$ in patient one (reference range in males: $22 \pm 6 \mathrm{~mL} \cdot \mathrm{m}^{-2}$ [6]). The stroke volume at rest was $35 \mathrm{~mL} \cdot \mathrm{m}^{-2}$ (reference range: $57 \pm 14 \mathrm{~mL} \cdot \mathrm{m}^{-2}$ ) and the heart rate at rest was 90 beats $\min ^{-1}$. The cardiac index at rest was low $\left(3.15 \mathrm{~L} \cdot \mathrm{min}^{-1} \cdot \mathrm{m}^{-2}\right)$. There were no signs of underlying cardiopathy. Heart magnetic resonance was performed in one case and confirmed the compression of the pulmonary vein.

Two of the patients had undergone previous treatment for sarcoidosis, but treatment had been discontinued for at least $1 \mathrm{yr}$ at the time of the left atrial compression diagnosis. Two patients were treated, but decision for therapy was not motivated by the left atrium compression. One patient received corticosteroids ( $1 \mathrm{mg} \cdot \mathrm{kg}^{-1}$ prednisone, gradually tapered) because of the rapid progression of the parenchymal lesions with severe functional impairment. Another patient was treated with infliximab (because of previous severe side-effects with corticosteroids, azathioprine and methotrexate) for incapacitating muscular involvement. In both cases, the size of the enlarged lymph nodes significantly decreased under therapy, and the left atrial compression disappeared (table 1).

To our knowledge, this is the first description of venoatrial compression in sarcoidosis. Many cases of compression of mediastinal structures by lymph nodes in sarcoidosis have been reported in the literature. Compression of pulmonary arteries by enlarged lymph nodes is considered to be a possible aetiology for pulmonary hypertension in sarcoidosis [4]. There is no real pathophysiological hypothesis for this unusual compressive nature.

When diagnosed with left atrial compression, all cases were reassessed, either for an alternative or for an additional diagnosis (sarcoidosis and cancer or lymphoma, sarcoidosis and tuberculosis, etc.). All sarcoidosis diagnosis had been made according to the ATS/ERS/WASOG statement [5], and there were no other atypical features apart from the compressive lymph nodes in any of the patients. New sputum samples were negative for tuberculosis. Tuberculin intradermal reaction remained negative. There was no clinical or radiological argument for cancer or malignant haemopathy. All patients were HIV negative. We ruled out histoplasmosis because of its extremely low prevalence in France. Last, we currently have 1-3 yrs of follow-up on these 
TABLE 1 Characteristics of venoatrial compression and pulmonary function at rest and on exercise

\begin{tabular}{|c|c|c|c|c|}
\hline & \multicolumn{4}{|c|}{ Patient } \\
\hline & 1 & $2^{\#}$ & $3^{\#}$ & 4 \\
\hline Subcarineal lymphadenopathy size $\mathrm{cm}$ & $7.5 \times 4.5$ & $8 \times 4.1$ & $6.4 \times 4.2$ & $4.07 \times 2.5$ \\
\hline Pulmonary vein compression & LSPV LIPV (and deviation of right pulmonary veins) & LSPV, LIPV & LSPV, LIPV & \\
\hline FVC $\%$ pred & 81 & 83 & 77 & 97 \\
\hline Ventilatory reserve \% pred & 26 & 32 & 23 & 37 \\
\hline Heart rate $\%$ pred & 91 & 71 & 80 & 91 \\
\hline Oxygen pulse \% pred & 54 & 57 & 66 & 69 \\
\hline $\mathrm{PA}_{\mathrm{A}-\mathrm{aO}}{ }_{2} \mathrm{mmHg}$ & 27 & 28.4 & 58 & 34 \\
\hline
\end{tabular}

LSPV: left superior pulmonary vein; LIPV: left inferior pulmonary vein; FVC: forced vital capacity; \% pred: \% predicted; $D L, C O$ : diffusing capacity of the lung for carbon monoxide; $V^{\prime} \mathrm{O}_{2}$ : oxygen uptake; $\mathrm{PA}_{\mathrm{a}} \mathrm{aO}$ : alveolo-arterial gradient for oxygen tension. ${ }^{*}$ : patient was taking $\beta$-blockers.

cases; two were resolved under corticosteroid or immunosuppressive treatment, and the two other showed no evolution. No new element that was not consistent with the diagnosis of sarcoidosis occurred.

The cases we report are all recent; therefore, we make the hypothesis that left atrial compression by enlarged lymph nodes in sarcoidosis may not be such a rare entity and is probably underdiagnosed, for two reasons. First of all, it is unclear whether a moderate left atrial compression induces symptoms, and if so, they would be nonspecific in the setting of a respiratory disease. In one of the few reviews addressing the issue, D'CRUZ et al. [7] distinguish different degrees in cardiac involvement of mediastinal masses. He describes a mediastinal mass as being "compressive" if it produces haemodynamic effects and symptoms akin to tamponade. On the contrary, a mass labelled as "encroaching on" the heart would narrow or distort it but would not produce haemodynamic effects or clinical symptoms. However, one cannot rule out that a moderate compression or encroachment on the left atrium would produce more subtle haemodynamic alterations and, therefore, symptoms, especially during exercise. Dyspnoea on exercise is reported in several cases of left atrial compression of other causes, such as bronchogenic cyst or hiatus hernia. Though none of our patients presented signs of tamponade or cardiac failure, all of them complained of dyspnoea on exertion and decreased tolerance to exercise. While two of the patients had moderate to severe alteration of carbon monoxide diffusion, the remaining two had a normal or close to normal oxygen saturation and oxygen alveolo-arterial gradient on exercise, as well as a preserved ventilatory reserve. In these patients, the oxygen pulse remained low throughout exercise, suggesting a limitation in the increase of stroke volume on exertion $[8,9]$ caused by the compression, which impairs the reservoir function of the left atrium. A low peak oxygen pulse was also found in the other two patients, but its interpretation was difficult due to oxygen desaturation on exercise, and possible muscular involvement. Pulmonary vein stenosis usually induces symptoms when several veins are involved and when the stenosis is $>60 \%$ [10], which was not the case for our patients. Furthermore, good visualisation of the left atrial and pulmonary vein compression on CT scan may require contrast, which is not routinely used in sarcoid patients. Even so, a visible compression of the left atrium may go unnoticed to the radiologist and the clinician alike, as well as to the echocardiographist, who do not expect such an abnormality. In two of our patients, the compression was already visible on at least one previous CT scan or echocardiography before it was eventually diagnosed. We recommend paying careful attention to the left atrial posterior wall and to the pulmonary veins in patients with large subcarinal lymph nodes.

The compression disappeared for the two patients that were treated, which is consistent with many reports about compression of bronchi or pulmonary arteries. It is uncertain whether a left atrial and/or pulmonary vein compression should represent an indication for treatment per se, as their clinical relevance and role in the onset of symptoms are unclear and difficult to assess. One must also bear in mind that such compressions may have a spontaneous positive outcome.

In conclusion, compression of various mediastinal structures by lymphadenopathy in sarcoidosis has already been described as an atypical form of the disease. This is, however, the first report of left atrial and pulmonary vein compression. Awareness about the existence of this condition, especially in patients who show large subcarineal lymphadenopathy, should increase its diagnosis, and allow us to understand better its possible influence on exercise capacity.

\section{E. Morawiec*, A-L. Hachulla-Lemaire", J. Chabrol*, M. Rémy-Jardin" ${ }^{\#}$ and B. Wallaert*}

*Clinique des maladies respiratoires, Centre de compétence des maladies pulmonaires rares, Hôpital Calmette, CHRU de Lille, and "Service d'imagerie thoracique, Hôpital Calmette, CHRU de Lille, Lille cedex, France.

Correspondence: B. Wallaert, Clinique des maladies respiratoires, Hôpital Calmette, CHRU 59037, Lille cedex, France. E-mail: Benoit.wallaert@chru-lille.fr

Statement of Interest: None declared. 
Acknowledgements: T. Le Tourneau, Service d'explorations fonctionnelles cardio-vasculaires, Hôpital cardiologique, CHRU de Lille, Lille cedex, France.

\section{REFERENCES}

1 Lynch JP 3rd, Computed tomographic scanning in sarcoidosis. Semin Respir Crit Care 2003; 24: 393-418.

2 Rockoff SD, Rohatgi PK. Unusual manifestations of thoracic sarcoidosis. AJR Am J Roentgenol 1985; 144: 513-528.

3 Bouvry D, Uzunhan Y, Naccache JM, et al. [Atypical sarcoidosis.] Rev Med Interne 2008; 29: 46-53.

4 Nunes H, Humbert M, Capron F, et al. Pulmonary hypertension associated with sarcoidosis: mechanisms, haemodynamics and prognosis. Thorax 2006; 61: 68-74.

5 ATS/ERS/WASOG statement on sarcoidosis. American Thoracic Society/European Respiratory Society/World Association of
Sarcoidosis and other Granulomatous Disorders. Sarcoidosis Vasc Diffuse Lung Dis 1999; 16: 149-173.

6 Lang RM, Bierig M, Devereux RB, et al. Recommandations for chamber quantification. Eur J Echocardiography 2006; 7: 79-108.

7 D'Cruz IA, Feghali N, Gross CM. Echocardiographic manifestations of mediastinal masses compressing or encroaching on the heart. Echocardiography 1994; 11: 523-533.

8 Jones N. Clinical Exercise Testing. 4th Edn. Philadelphia, WB Saunders, 1997.

9 ATS/ACCP Statement on cardiopulmonary exercise testing. Am J Respir Crit Care Med 2003; 167: 211-277.

10 Packer DL, Keelan P, Munger TM, et al. Clinical presentation, investigation, and management of pulmonary vein stenosis complicating ablation for atrial fibrillation. Circulation 2005; 111 546-554.

DOI: 10.1183/09031936.00189209 